\title{
Aspergilosis necrotizante crónica en un paciente con secuelas de tuberculosis pulmonar
}

\author{
ALCIDES ZAMBRANO F.*, ANDREA BIERE A.** y DIONIS ISAMITT D.***
}

Chronic necrotizing aspergillosis in a patient
with pulmonary tuberculosis sequelae

Aspergillus fumigatus is the causative agent of differents pathologies in the human being: aspergilloma, allergic bronchopulmonary aspergillosis, chronic necrotizing aspergillosis and invasive aspergillosis. In chronic necrotizing aspergillosis there is local invasion of the lung tissue and parenchyma destruction. Chronic necrotizing aspergillosis is different from invasive aspergillosis, because the abscence of vascular invasion or dissemination. Chronic necrotizing aspergillosis is seen in middle-aged and elderly with underlying lung diseases: COPD, tuberculosis sequelae, lung resection, pneumoconiosis, radiotherapy, lung infarction or sarcoidosis. Clinical manifestations are non specific, being the most usual fever, cough, sputum production and weight loss. Incidence of chronic necrotizing aspergillosis is unknown in Chile. Chronic necrotizing aspergillosis can produce death. It requires early diagnosis and treatment. In a patient with a predisposing disease and with prolonged fever and consuntive status, diagnosis of chronic necrotizing aspergillosis should be considered. We present a patient with chronic necrotizing aspergillosis attended at Instituto Nacional del Tórax (Thorax National Institute) in Santiago.

Key words: Pulmonary aspergillosis; tuberculosis; itraconazole.

\section{Resumen}

Aspergillus fumigatus puede causar diferentes patologías en el ser humano: aspergiloma, aspergilosis broncopulmonar alérgica, aspergilosis necrotizante crónica, aspergilosis invasora. En la aspergilosis necrotizante crónica hay invasión local del parénquima y destrucción. A diferencia de la aspergilosis invasora no invade vasos sanguíneos ni se disemina a otros órganos. La aspergilosis necrotizante crónica se presenta en pacientes de edad media o ancianos con patología pulmonar previa: EPOC, secuelas de tuberculosis, resección pulmonar, neumoconiosis, radioterapia, infarto pulmonar o sarcoidosis. La clínica es indolente e inespecifica, con fiebre, tos, expectoración y baja de peso. Se desconoce la incidencia de aspergilosis necrotizante crónica en nuestro medio. La aspergilosis necrotizante crónica es potencialmente fatal, por lo que requiere de un diagnóstico y tratamiento oportuno. Creemos que, debe considerarse esta entidad ante un cuadro consuntivo y febril prolongado, en pacientes con enfermedades predisponentes que producen una leve baja de la inmunidad. Describimos el caso de un paciente atendido en el Instituto Nacional del Tórax.

Palabras clave: Aspergilosis pulmonar; tuberculosis; itraconazole.

\footnotetext{
* Residente Enfermedades Respiratorias. Instituto Nacional del Tórax, Universidad de Chile.

** Residente de Medicina Interna, Hospital Clínico Universidad de Chile.

*** Médico broncopulmonar, Servicio Médico-Quirúrgico, Instituto Nacional del Tórax.
} 


\section{Introducción}

Aspergillus fumigatus es un hongo filamentoso que se encuentra ampliamente distribuido en el ambiente. Cuando sus esporas $(2$ a $3 \mu \mathrm{m})$ son inhaladas, se depositan en el pulmón y son capaces de causar diferentes patologías en el ser humano: aspergiloma, aspergilosis broncopulmonar alérgica, aspergilosis necrotizante crónica, aspergilosis invasora. Aunque éstas son entidades diferentes, en raras ocasiones pueden cambiar a otra, por ejemplo un aspergiloma puede evolucionar a aspergilosis invasiva ${ }^{1}$.

El aspergiloma es la forma más común de compromiso pulmonar por Aspergillus y se observa cuando se coloniza una cavidad preexistente en el pulmón, frecuentemente una secuela de tuberculosis. El aspergiloma es una masa de micelios, células inflamatorias, fibrina, restos de tejido, mucus, que conforman una bola fúngica dentro de la cavidad. Generalmente, es asintomático, pero puede causar hemoptisis como manifestación más frecuente. El diagnóstico se hace como hallazgo en un estudio radiológico solicitado por otros motivos o durante el estudio de una hemoptisis ${ }^{2}$.

La aspergilosis broncopulmonar alérgica (ABPA) se ve en aproximadamente el 14\% de los pacientes asmáticos severos y con menor frecuencia en pacientes con fibrosis quística. No se conoce bien la patogénesis de ABPA, pero hay evidencia de que sería una reacción de hipersensibilidad a los antígenos de A. fumigatus. Estos pacientes presentan infiltrados pulmonares recurrentes y eosinofilia, junto con elevación de $\mathrm{IgE}$ e IgG específicas. Destaca la presencia de bronquiectasias proximales, las que pueden faltar cuando el diagnóstico es precoz ${ }^{3,4}$.

Tanto el aspergiloma como ABPA son infecciones limitadas al pulmón, sin evidencia de invasión a los tejidos. En cambio en la aspergilosis invasora hay daño tisular e invasión de vasos pulmonares, con lo cual la infección puede diseminarse por el torrente sanguíneo, alcanzando otros órganos, como el sistema nervioso central, piel, riñón, hígado, etc. Generalmente, ocurre en pacientes inmunocomprometidos, siendo excepcional en inmunocompetentes. Las manifestaciones clínicas más comunes son fiebre, tos húmeda, disnea y en los casos de diseminación a distancia van a depender del órgano comprometido, como convulsiones, deterioro de la conciencia en el caso de compromiso del SNC. La letalidad, aún en nuestros días, permanece alta $^{5}$.

En la aspergilosis necrotizante crónica (ANC), también denominada aspergilosis semi-invasiva, hay una invasión local del parénquima, con destrucción y formación de cavidades. A diferencia del aspergiloma, no requiere la existencia de una cavidad, aunque en ocasiones a partir de la invasión local del tejido pulmonar adyacente a un aspergiloma se puede desarrollar el cuadro de ANC. Se distingue también de la aspergilosis invasora en que no hay invasión de vasos sanguíneos ni diseminación a otros órganos. ANC se ve generalmente en pacientes de edad media $\mathrm{o}$ ancianos que tienen una historia de EPOC, tuberculosis, resección pulmonar, neumoconiosis, radioterapia por una neoplasia o, con mucho menos frecuencia, fibrosis quística, infarto pulmonar o sarcoidosis, diabetes, uso prolongado de corticoides, condiciones que producen una inmunodepresión leve. El cuadro clínico es indolente, presentándose en el curso de varios meses fiebre, tos, expectoración y baja de peso ${ }^{5,6}$.

Como la ANC es una patología potencialmente fatal, requiere de un diagnóstico oportuno e instauración del tratamiento apenas este se confirme, lo cual puede evitar el deterioro progresivo en la condición del paciente ${ }^{5,6}$.

Se desconoce la real incidencia de ANC en nuestro medio. Después de hacer una revisión de la literatura, no encontramos trabajos publicados en revistas nacionales que revisen esta entidad nosológica.

Consideramos de importancia tener en consideración esta entidad ante un cuadro consuntivo $\mathrm{y}$ febril prolongado, en pacientes con las afecciones predisponentes antes mencionadas. A continuación describimos el caso de un paciente atendido en el Instituto Nacional del Tórax.

\section{Caso Clínico}

Paciente de 58 años que presenta como antecedentes el haber sido un gran fumador (índice paquetes/año de 40), hábito que suspendió hace cinco años; haber padecido una tuberculosis pulmonar hace aproximadamente diez años, por la cual no recibió tratamiento, evidenciándose sus lesiones secuelares en la radiología. El año 2003 es hospitalizado por hemoptisis, diagnosticándose en esa oportunidad un aspergiloma, motivo por el cual es sometido a una lobectomía superior izquierda.

Posterior a la cirugía resectiva de pulmón, inicia un cuadro lentamente progresivo de baja de peso (cuantificada en $14 \mathrm{~kg}$ ), asociado a anorexia y tos crónica productiva. Su asistencia a los controles ambulatorios postquirúrgicos es 
Tabla 1. Exámenes serológicos del paciente

\begin{tabular}{llcc}
\hline Examen & Hospitalización & $\begin{array}{c}\text { Primer control } \\
\text { (2 meses) }\end{array}$ & $\begin{array}{c}\text { Último control } \\
\text { (9 meses) }\end{array}$ \\
\hline $\begin{array}{l}\text { Anticuerpos IgG específicos } \\
\text { Aspergillus fumigatus }\end{array}$ & Positivo 0,676 UA & Positivo 0,619 UA & Positivo 0,573 UA \\
$\begin{array}{l}\text { Inmunoprecipitación (DDA) } \\
\text { Positivo } 1 \text { banda }\end{array}$ & Positivo 1 banda & Positivo 1 banda \\
\hline
\end{tabular}

irregular. Evaluado en forma ambulatoria, se realizan repetidas baciloscopías de expectoración (un total de seis) en las cuales no se observan bacilos alcohol ácido resistentes (BAAR), con cultivos en medio de Löwenstein Jensen negativos. La determinación de IgG mediante técnica de ELISA para Aspergillus es permanentemente positiva a título elevado (Tabla 1$)^{7}$. Las radiografías de tórax solicitadas, evidencian imágenes de infiltración pulmonar que progresan lentamente en el lóbulo inferior izquierdo remanente (Figura 1). Como el paciente refiere que la sintomatología se hace más marcada en las últimas semanas y el compromiso del estado general es mayor, se decide su ingreso al Servicio de Medicina del Instituto Nacional del Tórax, para continuar el estudio hospitalizado.

Al momento de su ingreso al Servico de Medicina el paciente se encuentra enflaquecido, con discreta palidez de mucosas. No presenta fie-

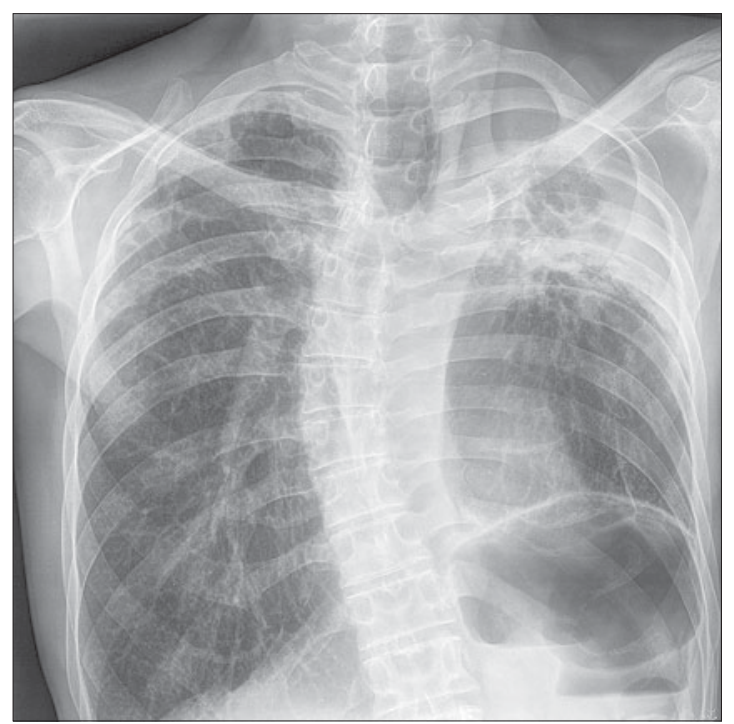

Figura 1. Radiografía de tórax de ingreso del paciente (mayo de 2005). Llama la atención las imágenes de opacidades y de pequeñas cavidades en el segmento apical del lóbulo inferior izquierdo remanente. bre. Su presión arterial es de 106/73 mmHg, presenta taquicardia de 105 pulsaciones por minuto y polipnea leve. No se palpan adenopatías. $\mathrm{Al}$ examen cardíaco no se pesquisan alteraciones y al examen pulmonar destacan crepitaciones aisladas y roncus espiratorios escasos. No se palpan hepato ni esplenomegalia.

En el laboratorio hay una discreta anemia microcítica normocrómica y eosinofilia leve; VHS de $40 \mathrm{~mm} / \mathrm{h}$; pruebas de función hepática, renal y tiroídea normales (Tabla 2).

TAC de tórax revela lesiones residuales postquirúrgicas y cicatriciales en el pulmón remanente izquierdo, bronquios dilatados, cavidades y opacidades con focos de relleno acinar; lesiones cicatriciales en el lóbulo superior derecho; hilios y mediastino normales (Figura 2). La fibrobroncoscopía no muestra alteraciones, describiéndose el muñón del bronquio lobar superior izquierdo con indemnidad de la mucosa. El estudio del lavado bronquioalveolar fue negativo para células neoplásicas, bacterias y BAAR. La ecotomografía abdominal no informa hallazgos de importancia y la endoscopía digestiva alta observa una mucosa del tracto digestivo normal.

Ante la evidencia de la presencia de $A$. fumigatus, los hallazgos radiológicos sugerentes y habiéndose descartado razonablemente otras causas de baja de peso y compromiso del estado general del paciente, se hace el diagnóstico de ANC de acuerdo a los criterios establecidos en la literatura y se inicia tratamiento antifúngico con itraconazol $400 \mathrm{mg} /$ día $^{1,5}$.

La respuesta clínica del paciente es favorable, con apetito conservado y sensación subjetiva de bienestar. El tratamiento con itraconazol es bien tolerado.

Tras 15 días de hospitalización, se indica su alta, para continuar tratamiento y control clínico y radiológico ambulatorio.

En el primer control, 2 meses posterior al alta, el paciente refiere sentirse mucho mejor, se encuentra asintomático y ha experimentado una discreta alza ponderal de $2 \mathrm{~kg}$. El examen físico 
Tabla 2. Principales exámenes de laboratorio realizados al paciente

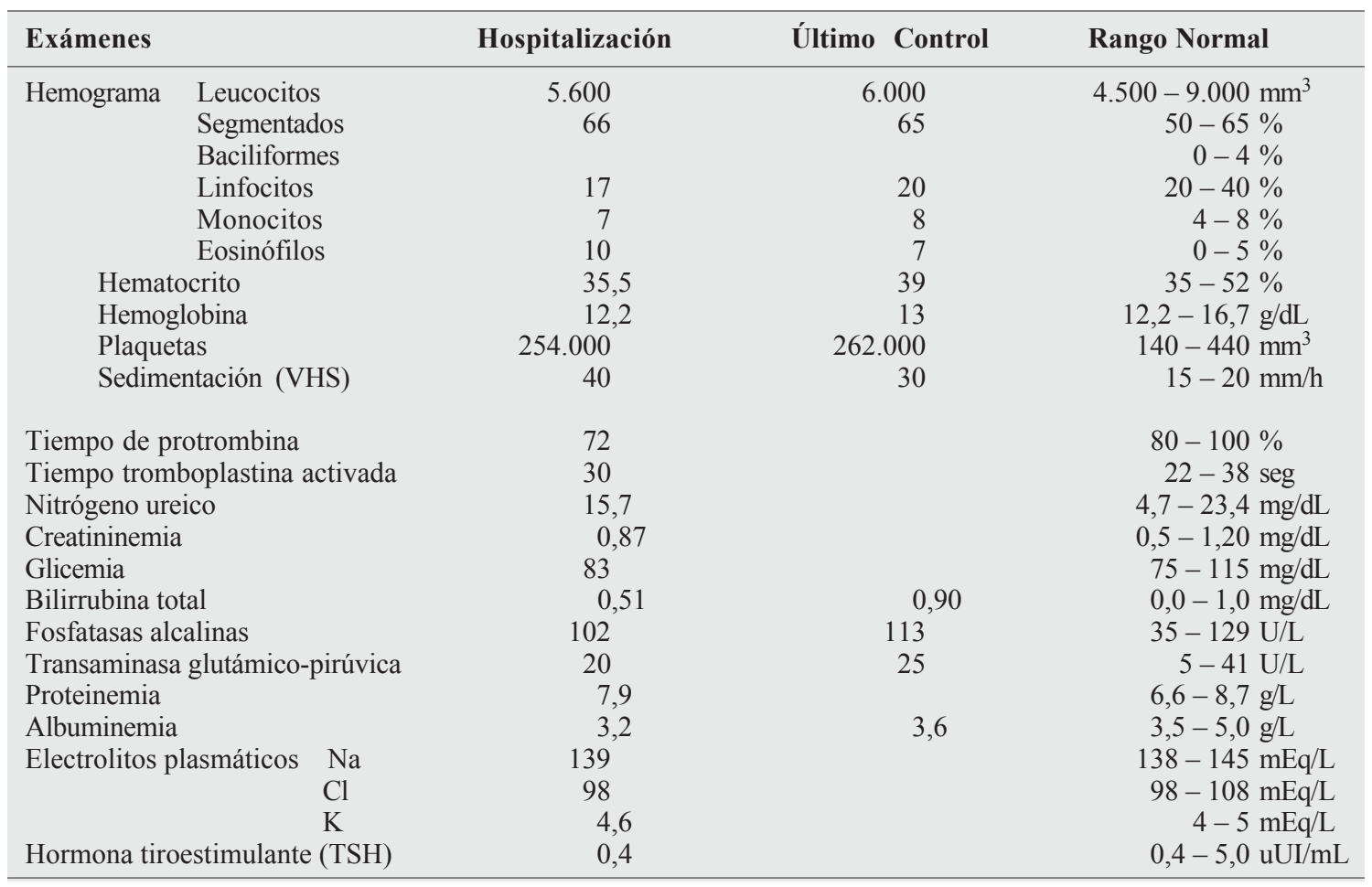

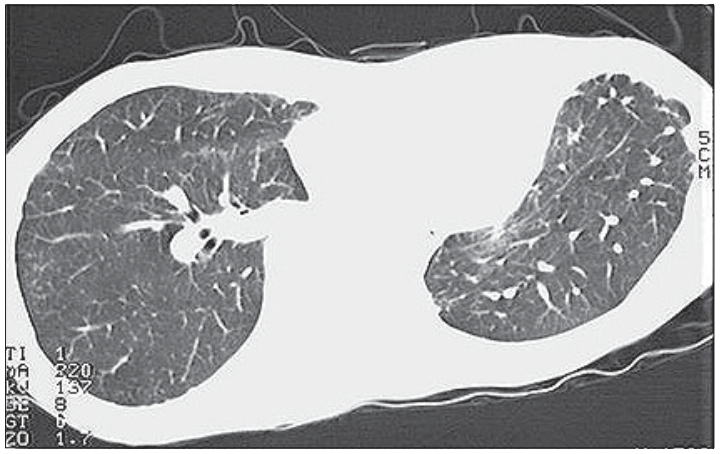

Figura 2. TAC de tórax (mayo de 2005). En el corte se observan bronquios de paredes engrosadas y opacidades mal definidas periféricas en lóbulo inferior izquierdo, asociadas a áreas de engrosamiento pleural (lesiones sugerentes de $\mathrm{ANC}$ ).

pulmonar es normal. La serología para $A$. fumigatus sigue siendo positiva, aunque a un título menor. Se observa discreta mejoría en algunos parámetros de laboratorio, como menor recuento de eosinófilos en sangre, disminución de la VHS $\mathrm{y}$ aumento del hematocrito. Hay normalización de la albuminemia (Tabla 2). El TAC de tórax evidencia regresión parcial de opacidades en lóbulo inferior izquierdo remanente (Figura 3 ).

A los 9 meses del alta el paciente se mantiene

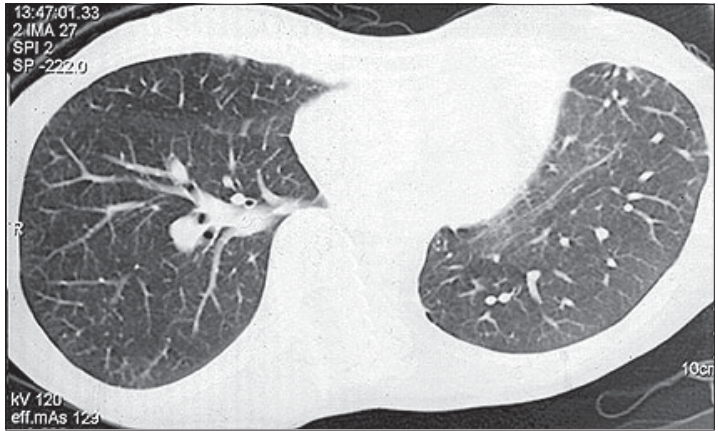

Figura 3. TAC de tórax de control a los 2 meses de tratamiento (julio de 2005). Nótese la regresión parcial de opacidades, especialmente periféricas y áreas de engrosamiento pleural, en lóbulo inferior izquierdo.

estable. Ha subido $5 \mathrm{~kg}$ de peso y se encuentra asintomático. Toleró bien el tratamiento con itraconazol oral, el cual se suspendió a los 7 meses.

\section{Discusión}

En 1981, se comunicó por primera vez un caso de ANC, en un paciente con una enfermedad pulmonar cavitaria crónica y posteriormente 
ha habido publicaciones de diferentes series de pacientes $^{8,9}$. Varios años después, en 2003, Denning et al, en un reporte de 18 casos, acuñaron el término aspergilosis pulmonar cavitaria crónica, para referirse a una entidad caracterizada por sintomatología de inicio y progresión lenta, donde destacaban la pérdida de peso, tos crónica, fatiga y dolor torácico; encontrándose cavidades pulmonares múltiples con o sin engrosamiento pleural y con o sin la presencia de aspergiloma ${ }^{10}$. Dicha descripción no difiere fundamentalmente de la definición de $\mathrm{ANC}$, por lo que creemos se trata de una única entidad ${ }^{8-10}$.

Si bien en la literatura nacional no hay reportes de ANC, en 1974, año en que aún no estaba descrita, Yarzábal y cols reportaron 25 pacientes con "aspergilosis intracavitaria" dentro de una serie de 48 casos con aspergilosis pulmonares, algunos de los cuales tuvieron formas de presentación que podrían corresponder a $\mathrm{ANC}^{11}$.

Como la clínica es inespecífica y se instala en el curso de semanas o meses, es fundamental la sospecha clínica para orientar el estudio diagnóstico. La radiografía de tórax es útil en el diagnóstico observándose en los lóbulos superiores o en los segmentos superiores de los lóbulos inferiores un infiltrado con cavidades. Es posible observar un aspergiloma concurrente en la mitad de los pacientes, el que presenta signos de invasión local, con engrosamiento de la pleura adyacente. TAC de tórax puede entregar información adicional ${ }^{12}$. El diagnóstico se certifica demostrando la presencia de hifas de Aspergillus en el cultivo. En general, no es necesario hacer estudios invasivos como biopsia pulmonar quirúrgica o videotoracoscopía, habiéndose desarrollado criterios de diagnóstico clínico: estudio radiológico sugerente, exclusión de otras condiciones que pueden presentar clínica similar (tuberculosis, micobacterios atípicos, histoplasmosis cavitaria crónica, coccidioidomicosis) y demostración de Aspergillus en cultivo. La determinación sérica de IgG anti antígenos de Aspergillus (por su alta especificidad para el género Aspergillus) y los test cutáneos específicos para Aspergillus son útiles como ayuda diagnóstica ${ }^{1}$.

ANC puede llevar a la muerte del paciente en un plazo variable (promedio de doce meses), por lo que una vez hecho el dignóstico debe instaurarse el tratamiento. Con el tratamiento antifúngico (Anfotericina B, itraconazol) la evolución es generalmente favorable, aunque el pronóstico a largo plazo no está bien documentado. En diferentes series de pacientes, la respuesta exitosa a la terapia endovenosa con anfotericina $\mathrm{B}$ (en dosis mayores a 1,5-2 gr) no va más allá del $40 \%$ a $50 \%{ }^{5}$. Aunque existe escasa experiencia, con el uso de anfotericina $\mathrm{B}$ intracavitaria $(0,5 \mathrm{~g})$ administrada en forma percutánea, se han logrado buenos resultados en cerca del $80 \%$ de los $\operatorname{casos}^{13}$. Los principales inconvenientes de la anfotericina B son su mala tolerancia y sus reacciones adversas severas ${ }^{14}$. En relación al tratamiento con itraconazol (400 mg/día administrado por 1 a 18 meses), se reportan mejoría o cura hasta en el $90 \%$ de los pacientes, aunque se ha descrito recurrencia al discontinuar la terapia $^{5,15}$. DeBeule et al comunicaron la mejoría clínica de 39 de 44 pacientes $(88,6 \%)$ tratados con itraconazol por 1 a 18 meses $^{16}$. En una serie de 12 pacientes con ANC, Dupont encontró cura o mejoría en $11(91,6 \%)$ al tratarlos por 1 a 7 meses con itraconazol, aunque 3 recayeron al discontinuar el antifúngico ${ }^{17}$. En una pequeña serie de 3 pacientes, se reportaron recurrencias del cuadro de ANC al suspender la terapia con itraconazol, a pesar de una buena respuesta clínica inicial ${ }^{18}$. Itraconazol es más efectivo que anfotericina $\mathrm{B}$, tiene baja toxicidad y es mejor tolerado $^{5,15}$. Nuevas drogas antifúngicas, como el voriconazol, son activas contra Aspergillus y son promisorias en el tratamiento de ANC, aunque su alto costo ha restringido su mayor empleo $^{19}$.

En nuestro paciente el diagnóstico de ANC está bien establecido por: 1) presencia de infección por $A$. fumigatus demostrada por la resección de un aspergiloma; 2) serología específica (IgG anti Aspergillus fumigatus persistentemente elevada; 3) lesiones destructivas locales con cavitación características en la radiografía y TAC de tórax; 4) cuadro clínico concordante con un proceso consuntivo prolongado; 5) descarte de otras causas de baja de peso y fiebre; 6) respuesta aceptable a la terapia antifúngica empleada, con una mejoría significativa en su condición, tanto clínica como radiológica ${ }^{1}$.

El hecho de que no haya comunicaciones previas en la literatura nacional sobre esta entidad nosológica, abre la posibilidad de que exista un subdiagnóstico en nuestro medio. Creemos que es de vital importancia tener presente esta patología para hacer un reconocimiento oportuno y evitar un desenlace fatal, pues se cuenta con tratamientos seguros y efectivos.

\section{Bibliografía}

1.- SOUBANI A O, CHANDRASEKAR P H. The clinical spectrum of pulmonary aspergillosis. Chest 2002; 121: 1988-99.

2.- KAWAMURA S, MAESAKI S, TOMONO K, 
TASHIRO T, KOHNO S. Clinical evaluation of 61 patients with pulmonary aspergillosis. Intern Med 2000; 39: 209-12.

3.- CARRASCO E, SEPÚLVEDA R, ROJAS L, MÉNDEZ P, VICHERAT L, OTERO $\mathrm{M}$, et al. Perfil clínico inmunológico de la aspergilosis broncopulmonar alérgica (ABPA). Enf Respir Cir Torac 1988; 4: 7682.

4.- CARRASCO E. Aspergilosis broncopulmonar alérgica. Complicaciones poco usuales de la afección. Rev Chil Enf Respir 2004; 20: 30-6.

5.- SARACENO J L, PHELPS D T, FERRO $\mathrm{T} J$, FUTERFAS R, SCHWARTZ B D. Chronic necrotizing pulmonary aspergillosis: approach to management. Chest 1997; 112: 541-8.

6.- PARRA I, REMACHA A, REZUSTA A, SUÁREZ D, SUÁREZ J, HERRERAS J A, et al. Chronic necrotizing pulmonary aspergillosis. Med Mycol 2004; 42: 36971.

7.- SEPÚlVEDA R, LONGBOTTOM J L, PEPYS J. Enzyme linked immunosorbent assay (ELISA) for IgG and IgE antibodies to protein and polysaccharide antigens of Aspergillus fumigatus. Clin Allergy 1979; 9: 359-71.

8.- GEFTER W B, WEINGRAND T R, EPSTEIN D M, OCHS R H, MILLER W T. "Semi-invasive" pulmonary aspergillosis: a new look at the spectrum of Aspergillus infections of the lung. Radiology 1981; 140: 313-21.

9.- BINDER R E, FALING L J, PUGATCH R D, MAHASAEN C, SNIDER G L. Chronic necrotizing pulmonary aspergillosis: a discrete clinical entity. Medicine (Baltimore) 1982; 61: 109-24.

10.- DENNING D W, RINIOTIS K, DOBRASHIAN R, SAMBATAKOU $\mathrm{H}$. Chronic cavitary and fibrosing pulmonary and pleural aspergillosis: case series, proposed nomenclature change, and review. Clin Infect Dis 2003; 37 Supl 3: S265-80.
11.- YARZABAL L, SEPÚLVEDA R, RETAMAL C, KINZEL R, CASTRO M, SALAMANCA L. Pulmonary aspergillosis (author`s transl). Rev Méd Chile 1974; 102: 772-8.

12.- FRANQUET T, MÜLLER N L, GIMÉNEZ A, GUEMBE P, DE LA TORRE J, BAGUE S. Spectrum of pulmonary aspergillosis: histologic, clinical and radiologic findings. Radiographics 2001; 21: 825-37.

13.- HARGIS J L, BONE R C, STEWART J, RECTOR N, HILLER F C. Intracavitary amphotericin B in the treatment of symptomatic pulmonary aspergillosis. Am J Med 1980; 68: 389-94.

14.- GALLIS H A, DREW R H, PICKARD W W. Amphotericin B: 30 years of clinical experience. Rev Infect Dis 1990; 12: 308-29.

15.- SCHIRALDI G F, GRAMEGNA G, DE ROSA C, LO CICERO S, CAPONE P, FERRARESE M, et al. Chronic pulmonary aspergillosis: current classification and therapy. Curr Opin Investig Drugs 2003; 4: 18691.

16.- DEBEULE K, DE DONCKER P, CAUWENBERGH G, KOSTER M, LEGENDRE R, BLATCHFORD N, et al. The treatment of aspergillosis and aspergilloma with itraconazole: clinical results of an open international study (1982-1987). Mycoses 1988; 31: 476-85.

17.- DUPONT B. Itraconazole therapy in aspergillosis: study in 49 patients. J Am Acad Dermatol 1990; 23: 607-14.

18.- CARAS W E, PLUSS J L. Chronic necrotizing pulmonary aspergillosis: pathologic outcome after itraconazole therapy. Mayo Clin Proc 1996; 71: 2530.

19.- HERBRECHT R, DENNING W, PATTERSON T F, BENNETT J E, GREENE R E, OESTMANN J W, et al. Voriconazole versus amphotericin B for primary therapy of invasive aspergillosis. N Engl J Med 2002; 347: 408-15.

Correspondencia a:

Alcides Zambrano F.

Teléfono: 098443662

Instituto Nacional del Tórax

E-mail: alcidedu@yahoo.es 